\title{
Krise durch Unterlassungen
}

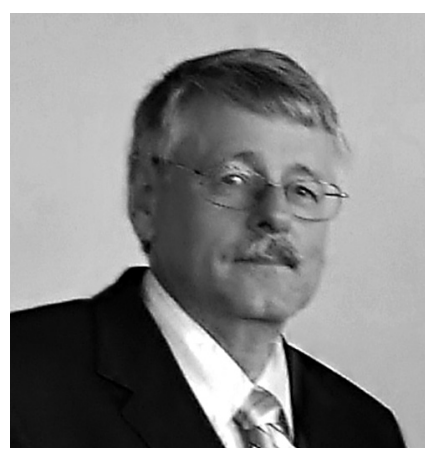

BERTHOLD BECHER

Dr. Berthold Becher war bis zu seiner Pensionierung Abteilungsleiter bei der Bank für Sozialwirtschaft AG. Seither ist er als Berater und Publizist in der Sozial- und Gesundheitswirtschaft tätig. bertholdbecher@aol.com

\author{
Nicht die demografische Entwicklung trägt die \\ Hauptschuld, wenn es in Organisationen und \\ Unternehmen zu einem Personalmangel kommt. \\ Oft sind es branchen- und hausinterne Versäumnisse, \\ die nun Anlass sein können, grundsätzlich über \\ künftige Geschäftsmodelle nachzudenken.
}

Glauben Sie immer noch daran, dass für die aktuellen Probleme bei der Rekrutierung von Personal in der Sozial- und Gesundheitswirtschaft der demografische Wandel die Ursache ist? Glauben Sie immer noch, dass langfristig der Import von Pflegekräften aus aller Herren Länder der relevante Beitrag zur Lösung auftretender Personalengpässe ist?

Hoffentlich nicht! Denn die Konsequenz wäre, dass Sie aufgrund einer unzutreffenden Ursachenerklärung sowie falscher Lösungswege die wichtigsten Ansatzpunkte zur Sicherung des Personals verkennen und daher nicht das richtige Tun bzw. dieses unterlassen. Denn sehen wir einmal von regionalen Besonderheiten ab: Generell gibt es zurzeit in der Sozial- und Gesundheits- wirtschaft auf dem Arbeitsmarkt zu verbessern und Beschäftigtenpotenzial zu heben.

Um den jetzigen und zukünftigen $\mathrm{He}$ rausforderungen zu entsprechen, müssen Unternehmen der Sozial- und Gesundheitswirtschaft die Beschäftigungsverhältnisse, die Arbeitsbedingungen, die Beschäftigtenstruktur, aber auch die Leistungsprozesse und die Angebotsstruktur sind zu überprüfen.

Angesichts des wirtschaftlichen Drucks durch Vergütungshöhe und Wettbewerb laufen Unternehmen Gefahr, sich auf solche personalwirtschaftlichen Maßnahmen zu fixieren, die in eine Rationalisierungsfalle führen: Nachhaltig negative wirtschaftliche Effekte treten auf wie beispielsweise gerin-

\section{"Neben dem Personalmanagement müssen die Leistungsprozesse und die Angebotsstruktur in den Blick genommen werden«}

wirtschaft keinen Personalmangel, der hauptsächlich bedingt wäre durch den veränderten Altersaufbau der Bevölkerung. Die aktuelle Situation ist Ergebnis von Unterlassungen: Unternehmen, Verbände, Branche, Politik und Finanzierungsträger haben sich bislang nicht hinlänglich bemüht, die relative Attraktivität der Sozial- und der Gesundheits- gere Attraktivität auf dem Arbeitsmarkt und auf dem Dienstleistungsmarkt sowie geringere Produktivität. Unternehmen, die einem entsprechenden Leitbild verpflichtet sind, müssen klären, wie ihre Maßnahmen des Personalmanagements dem eigenen Anspruch an die Beschäftigungsverhältnisse und die Leistungserbringung genügen. 


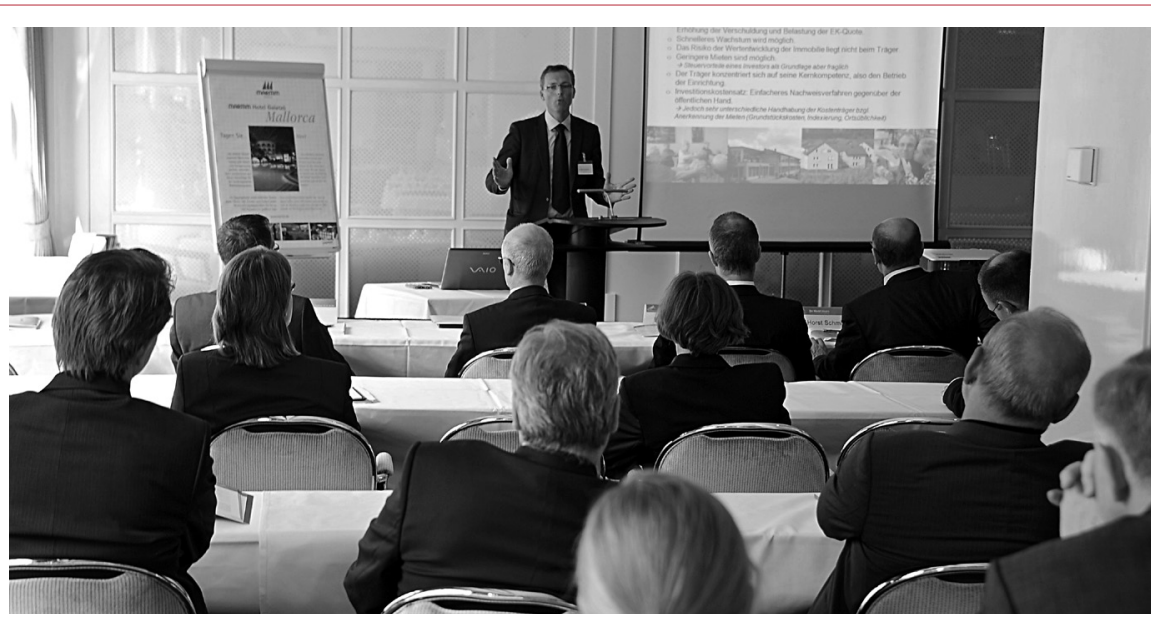

Die Frage, wie Verbände und Unternehmen der Sozialwirtschaft auf den Personalmangel reagieren sollten, diskutieren Vorstände und Geschäftsführer gemeinnütziger und privat-gewerblicher Unternehmen in einem Forum des 8. Kongresses der Sozialwirtschaft. Referenten aus der Branche werden dabei ihre Geschäftsstrategien und Maßnahmen zur Diskussion stellen, mit denen sie mittelfristig der Entwicklung auf den Arbeitsmarkt begegnen wollen. Besonders interessant sein wird zu erfahren, wie die eingeladenen Vertreter der Leistungsträger die personalwirtschaftlichen Herausforderungen sehen und welche Vorstellungen sie von deren Bewältigung haben.

www.sozkon.de

Die von den Entwicklungen auf dem Arbeitsmarkt und den übrigen wirtschaftlichen Rahmenbedingungen angestoßenen Veränderungen des Personalmanagements, gegebenenfalls auch des Leistungsangebotes und der Angebotsstruktur sind nicht in jedem Fall negativ zu bewerten. Sie können zu Veränderungen führen, die von den Unternehmen selbst, von Mitarbeitenden, Kunden und Finanzierungsträgern positiv bewertet werden.

Den personellen Herausforderungen aufgrund der Bedarf- und Arbeitsmarktentwicklung ist nicht allein mit binnenorganisatorischen Aktivitäten beizukommen. In den Blick zu nehmen sind auch die externen Restriktionen, denen sich Unternehmen bei den entsprechenden Gestaltungsmaßnahmen gegenübersehen. So hat beispielsweise die Entwicklung der Zuwendungs- und Leistungsfinanzierung ebenso Konsequenzen für ihre Handlungsoptionen angesichts des Arbeitsmarktes wie der Wettbewerb als Steuerungsmechanismus zur Reduzierung der Finanzierungsaufwendungen. Die Anbieter müssen daher prüfen, ob sie die vorhandenen Rahmenbedingungen als unausweichlich gegeben hinnehmen und nur anpassend reagieren unter Inkaufnahme größer werdender personeller und wirtschaftlicher Probleme oder ob sie mit ihren Verbänden konsequent auf die Veränderung der Rahmenbedingun- gen von Arbeitsmarkt, Finanzierung und

Die Bewältigung der jetzigen und zukünftigen personalwirtschaftlichen Herausforderungen der Sozial- und Gesundheitswirtschaft setzt eine geschäftspolitische und geschäftsstrategische Verankerung der Thematik in den Unternehmen voraus. Warum?

- Bei der Bewältigung des Personalproblems geht es mittlerweile um die Sicherung der Ertragskraft und Zukunftsfähigkeit der Unternehmen.

- Statt Personalverwaltung ist ein umfassendes, konzeptionell fundiertes und professionelles Personalmanagement mit entsprechender organisatorischer Ausgestaltung und geschäftspolitischer Verankerung notwendig.

- Den Herausforderungen ist gegebenenfalls nicht allein mit Personalmanagement zu begegnen. Mit in den Blick genommen werden muss auch die Neugestaltung der Leistungsprozesse und der Angebotsstruktur.

- Kooperationen mit anderen Unternehmen - im Verband und aus der Branche - können angebracht sein.

- Die Unternehmen und deren (Branchen-) Verbände müssen Einfluss nehmen auf die Rahmenbedingungen, die das Angebot auf dem Arbeitsmarkt und ihre Nachfragerposition beeinflussen. Wettbewerb hinwirken.
Die Sicherung der personellen Ressourcen für die Sozial- und die Gesundheitswirtschaft setzt auch voraus, dass zustande kommt, was bislang vernachlässigt worden ist: Einen "Pakt « aller relevanten Akteure wie Anbieter und ihre Verbände, Politik, Finanzierungsträger etc. Die Herausforderungen können wirksam nur abgestimmt und vorausschauend angegangen werden. Dies wurde bislang versäumt. Ohne eine solche konzertierte Aktion wird die Sicherung der personellen Ressourcen der Sozialund der Gesundheitswirtschaft in dem zunehmenden Wettbewerb auf dem Arbeitsmarkt sich zu einem noch größeren Problem auswachsen.

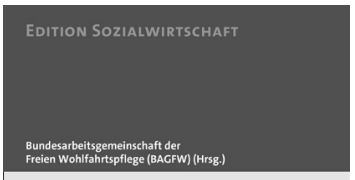

Den Wandel steuern. Personal und Finanzen als Erfolgsfaktoren

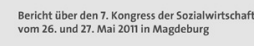

Y. Nomos

\section{Den Wandel steuern}

Die Sozialwirtschaft in Deutschland ist zwar ohne größere Blessuren durch die letzte Finanz- und Wirtschaftskrise gekommen. Doch nun stehen Wohlfahrtsverbände und soziale Unternehmen vor großen Herausforderungen, die es im Interesse ihrer Nutzer und Mitarbeitenden zu bewältigen gilt. Die Finanznöte von Kommunen und der Sozialversicherungen zählen ebenso zu den Klippen, die es zu umschiffen gilt, wie der demografische Wandel und die zunehmende Individualisierung und Komplexität der Angebote und Dienstleistungen - mit einem steigenden Bedarf an Transparenz von Angebot und Leistungserbringung. Der 7. Kongress der Sozialwirtschaft widmete sich vor zwei Jahren einer differenzierten Analyse der Probleme und der Suche nach funktionierenden Lösungen. Ein Sammelband vereinigt nun die Vorträge und Präsentationen dieses Branchentreffs.

Bundesarbeitsgemeinschaft der Freien Wohlfahrtspflege (Hg.): Den Wandel steuern. Personal und Finanzen als Erfolgsfaktoren. Nomos Verlagsgesellschaft, Baden-Baden 2012. 391 Seiten. 69,- Euro. ISBN 978-3-8329-6837-3. 